\title{
Milli-Tesla NMR and Spectrophotometry of Liquids Hyperpolarized by Dissolution Dynamic Nuclear Polarization
}

\author{
Yue Zhu, ${ }^{\dagger}$ Chia-Hsiu Chen, ${ }^{\dagger}$ Zechariah Wilson, ${ }^{\dagger}$ Igor Savukov ${ }^{\ddagger}$ and Christian Hilty ${ }^{\dagger}{ }^{*}$ \\ ${ }^{\dagger}$ Chemistry Department, Texas A\&M University, 3255 TAMU, College Station, TX 77843, USA \\ ${ }^{*}$ New Mexico Consortium, 100 Entrada Drive, Los Alamos, NM 87544, USA \\ *e-mail: chilty@tamu.edu
}

\begin{abstract}
Hyperpolarization methods offer a unique means of improving low signal strength obtained in lowfield NMR. Here, simultaneous measurements of NMR at a field of $0.7 \mathrm{mT}$ and laser optical absorption from samples hyperpolarized by dissolution dynamic nuclear polarization (D-DNP) are reported. The NMR measurement field closely corresponds to a typical field encountered during sample injection in a D-DNP experiment. The optical spectroscopy allows determination of the concentration of the free radical required for DNP. Correlation of radical concentration to NMR measurement of spin polarization and spin-lattice relaxation time allows determination of relaxivity and can be used for optimization of the D-DNP process. Further, the observation of the nuclear Overhauser effect originating from hyperpolarized spins is demonstrated. Signals from ${ }^{1} \mathrm{H}$ and ${ }^{19} \mathrm{~F}$ in a mixture of trifluoroethanol and water are detected in a single spectrum, while different atoms of the same type are distinguished by $J$-coupling patterns. The resulting signal changes of individual peaks are indicative of molecular contact, suggesting a new application area of hyperpolarized low-field NMR for the determination of intermolecular interactions.
\end{abstract}




\section{Keywords}

Low-field NMR, Dissolution Dynamic Nuclear Polarization, Relaxometry, Polarimetry

\section{TOC Figure}

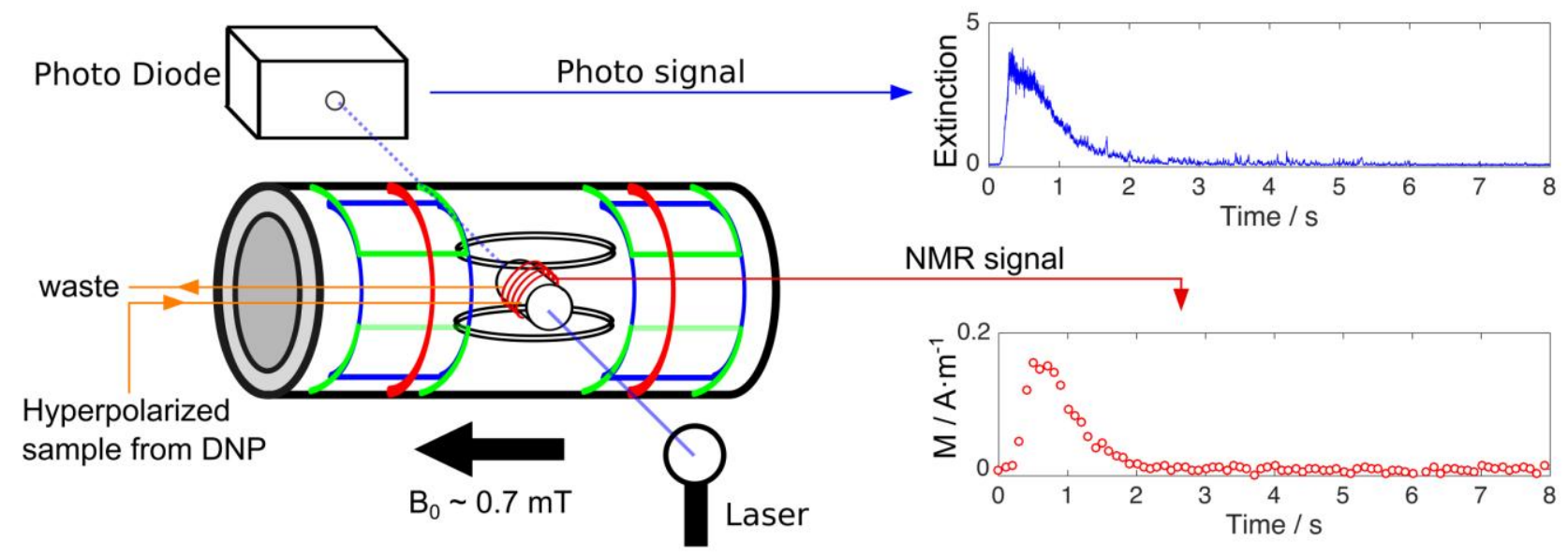




\section{Introduction}

Low-field (LF) NMR holds the promise of solving a specific set of problems where the use of high fields is impossible or impractical, or where aspects of spin physics result in new information. Applied to in vivo magnetic resonance imaging (MRI), LF detection not only represents a low-cost modality, but also provides enhanced relaxation contrast [1]. At LF, the penetration depth of radio-frequency radiation in metals is increased, reducing imaging artifacts and facilitating MRI in the presence of metallic structures. In NMR spectroscopy, relaxation dispersion measurements can be used to obtain information on molecular dynamics on large time scales [2-4]. Spin relaxation measurements at low field have further been used in remote locations for the characterization of geological samples or even ice cores [5,6]. Although chemical shifts are collapsed at fields below $\sim 2 \mathrm{mT}$, it is still possible to observe and correlate nuclei of different types using J-couplings [7]. Line widths typically are narrower than at higher field, which enables resolving J-couplings when they are otherwise unobservable $[8,9]$. J-spectroscopy even at zero-field has been shown to contain chemical information alternative to the traditional spectra based on chemical shifts at high fields $[10,11]$.

A major challenge in the application of LF NMR and MRI lies in the low obtainable signal strength compared to high-field NMR. The limitation in signal is first of all due to much smaller polarization, which is approximately proportional to the field strength $B_{0}$. In addition, the signal falls off as $B_{0}{ }^{1 / 2}$, when it can be reasonably assumed that Q-factors of the high- and low-frequency detection coils are comparable [12]. This latter sensitivity penalty at low field can be avoided by using ultrasensitive non-inductive detectors, such as superconductive quantum interference devices and atomic magnetometers [13-16]. However, this is not sufficient for most applications, and LF NMR methods, especially at the extreme end of ultra-low field, rely on pre-polarization (switching on/off a stronger magnetic field and shuttling a sample through a region of higher field [17]) as well as hyperpolarization 
techniques. Specifically, after hyperpolarization, the sensitivity of low field NMR or MRI can be as good as or better than than the signal obtained at high field [18]. Some examples of hyperpolarization in low field NMR include optically hyperpolarized xenon used for void space imaging, capitalizing on the ability of the low-frequency radiation to penetrate a metal enclosure [19], or Overhauser effect dynamic nuclear polarization (O-DNP) in the liquid state to image plants [20] and other samples [2124].

On the other hand, dissolution DNP (D-DNP), which over the past decade has become established as a versatile method for generating high polarization levels in liquid state NMR for many nuclei and chemical compounds, was not used in LF NMR. Thus in this paper, we propose and demonstrate the use of D-DNP as an alternative means for polarization enhancement in milli-tesla NMR. As an immediate application, we apply D-DNP LF NMR to determine the polarization level and spin-lattice relaxation times of hyperpolarized samples containing free radicals under typical conditions encountered in D-DNP. In addition, we demonstrate nuclear Overhauser effect (NOE) measurements that can be used for the determination of intermolecular interactions.

\section{Materials and Methods}

Experimental configuration. The experimental setup for LF NMR and optical measurements of DDNP samples is schematically depicted in Figure 1. The LF NMR apparatus consists of an electromagnet (a tetra-coil [25]) to produce the main NMR field $B_{0}$ in the horizontal direction, a set of shim coils to cancel external gradients and to enable imaging, and two orthogonal rf coils to excite and detect nuclear spins (Figure 1a). The current for generating the $B_{0}$ field was provided by a digitally regulated linear power supply (XDL35-5TP, Xantrax, Elkhart, IN). The shim coils, generating linear gradients in the $\mathrm{x}, \mathrm{y}$ and $\mathrm{z}$-directions, were positioned concentrically inside the tetra-coil. Shim currents were 
delivered using a dedicated shim power supply (Bruker Biospin, Billerica, MA). An aluminum shield between the shim coils and the rf coils was added to the setup to reduce electromagnetic interference from the environment. Separate sets of radio-frequency coils were used for NMR excitation and detection (Figure 1b) to reduce cross talk. The coils and flow cell were mounted on an insert printed from acrylonitrile butadiene styrene (ABS) plastic (Replicator 2X, MakerBot, Brooklyn, NY). The excitation coil was configured as Helmholtz pair ( $r=45 \mathrm{~mm}, n=15+15$ turns) to produce a vertical field. This coil was driven directly by the output of a data acquisition board (PCIe-6259, National Instruments, Austin, TX). The detection coil wound around the flow cell (242 turns) was connected to a metallized polyester film capacitor to form a resonance circuit with a $\mathrm{Q}$ factor of 29 . This resonant circuit was connected to the input of a pre-amplifier with adjustable gain and band-pass filter (SR-560, Stanford Research Systems, Sunnyvale, CA). After amplification, the signal was sent to the computer via data acquisition board for further signal processing. The flow cell was manufactured from Pyrex glass, fused with optical quality windows on both sides, for laser absorption measurements (Precision Glassblowing of Colorado, Centennial, CO).

For optical spectroscopy, a 3.5-mW laser with wavelength of 405-nm (Thorlabs, Newton, NJ) was mounted on a narrow optical bench attached below the electromagnet. Optical signal was detected using a photodiode (SMR1, Thorlabs) connected to a preamplifier built using a low-noise operational amplifier (OPA4131PJ, Texas Instruments, TX).

For hyperpolarization, the setup contained a HyperSense DNP polarizer (Oxford Instruments, Abingdon, UK). Dissolved samples were delivered through tubing (Teflon, 1/8" outer diameter, 1/16" inner diameter) to a sample injection valve (Figure 1c), where they were loaded into a 3.0-mL loop. The configuration of the injection valve was similar to that described in ref. [26]. Briefly, the completion of sample loading was sensed by an optical detector located at the outlet of the loop, which initiated switching of the injection valve (10W-0154L, VICI Valco, Houston, TX). A supply of water 
pressurized with nitrogen gas was then used for injecting the sample into the flow cell. The entire apparatus, including sample injection, pulse generation and data acquisition, was controlled using a LabView program (National Instruments, Austin, TX) [27].

(a)

(b)

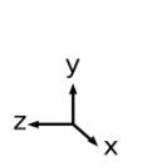

)
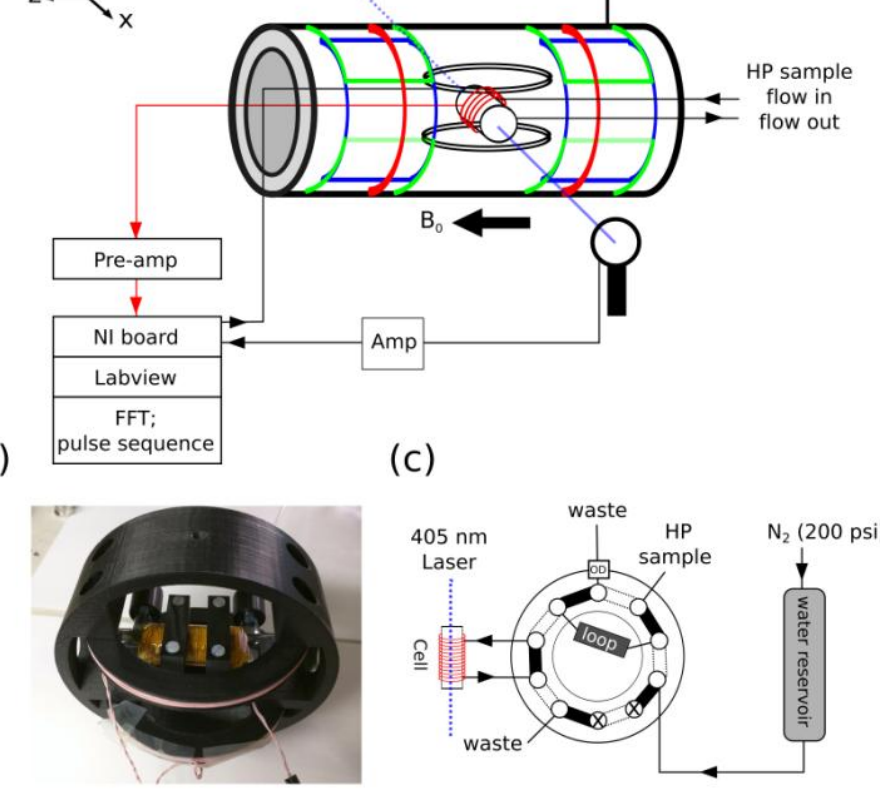

(c)

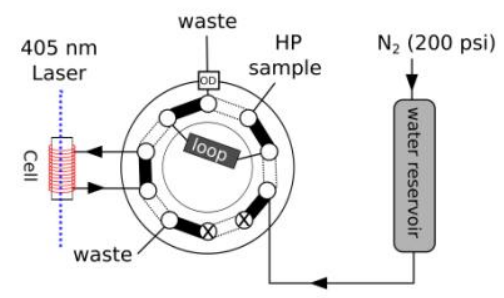

Figure 1: a) Configuration of the experiment. The flow cell, a small cylinder with the detection coil wound on its outer surface, is positioned in the center for simultaneous NMR and optical measurements. The excitation coil consists of two rings forming a Helmholtz arrangement with their axes perpendicular to that of the detection coil. Coils for shimming along three perpendicular axes $x, y$ and $z$ are wound on the large cylinder, and are drawn in blue, green and red, respectively. The laser beam intensity after the flow cell is measured with a photo-diode to monitor sample absorption. b) Geometry of core part of NMR spectrometer, including the flow cell with detection coil and excitation coil. c) Detail of the apparatus for sample transfer, consisting of an injection valve, a sample loop, and a reservoir containing pressurized water. The liquid flows in a first, "loading" mode (black bar) from the HP sample inlet into the loop. Subsequently, the injection valve switches to an "injection" position (dashed bar), causing pressurized water from the water reservoir to push the sample from the loop into the cell [26].

Hyperpolarization. Samples for ${ }^{1} \mathrm{H}$ measurement were prepared by dissolving $15-60 \mathrm{mM} 4$ Hydroxy-TEMPO (TEMPOL) in a mixture of $\mathrm{H}_{2} \mathrm{O} /$ ethylene glycol at 40/60 (v/v \%) to produce a glass-forming matrix. The sample for ${ }^{19} \mathrm{~F}$ measurement was prepared with $15 \mathrm{mM}$ TEMPOL in 
trifluoroethanol. Samples were loaded into the variable temperature insert of the DNP polarizer, where they were held at a temperature of $1.4 \mathrm{~K}$ and irradiated with microwaves for $20 \mathrm{~min}$ at a frequency of 94.005 GHz and power of $100 \mathrm{~mW}$. Subsequently, the samples were dissolved in a stream of $7.5 \mathrm{~mL}$ water, which had previously been heated in a closed vessel until a pressure of 10 bar was reached. The samples were taken into the loop of the injection device and driven into the flow cell using a pressure of 14 bar. For NMR measurements with stationary samples, the flow was stopped by simultaneous closure of inlet and outlet tubing. The injection valve was switched back to the original position following a pre-determined injection time of $700 \mathrm{~ms}$. For experiments detecting flow, the valve was not switched back to the original position.

NMR Spectroscopy. The ${ }^{1} \mathrm{H}$ nuclei were detected in a static magnetic field of $0.7328 \mathrm{mT}(1.899 \mathrm{~A}$ tetra-coil current) using thirty-five $31.2 \mathrm{kHz}$ small flip angle $\pi / 50.5$ pulses (each $0.16 \mathrm{~ms}$ ) with the interval duration being $0.3 \mathrm{~s}$. Signal acquisition was eight-fold oversampled. The signal was processed with a digital filter with high frequency cut off of $33000 \mathrm{~Hz}$ and low frequency cut off of $30000 \mathrm{~Hz}$. For determination of $T_{1}$, the spectrum obtained by performing Fourier transform on the time domain data was integrated to obtain the NMR amplitude for a given time. The spin-lattice relaxation time $T_{1}$ was found by fitting the amplitudes by the equation $a \cdot e^{-R t}+c$. with $R=R_{1}+\ln (\cos \theta) / \tau$ to take into account the effects of multiple spin excitations; here, $R_{1}$ is the spin-lattice relaxation rate, $\theta$ is the flip angle and $\tau$ is the time interval between pulses [28]. In the present experiments, the contribution due to the RF pulses was negligibly small, $7 \cdot 10^{-3} \mathrm{~s}^{-1}$, therefore $\mathrm{R}$ was equated to $\mathrm{R}_{1}$ without losing accuracy. For quantification of the paramagnetic relaxation enhancement (PRE), the obtained relaxation rates $R_{1}$ were fitted to the equation

$$
R_{1}=R_{1}^{0}+\varepsilon c
$$


where $R_{1}{ }^{0}$ is the extrapolated relaxation rate in the absence of radical, $\varepsilon$ is the paramagnetic relaxivity and $c$ is the radical concentration.

In the imaging experiment, the $x$-gradient, together with the others tuned to compensate external gradients, was partially removed to introduce a frequency encoding gradient in the x direction, $G_{x}$. The gradient strength was estimated as $G_{\mathrm{x}}=1.71 \cdot 10^{-4} \mathrm{mT} / \mathrm{cm}$ from the full width at half maximum of the signal spectrum and the detection coil length. Multiple delay times at $t=5,10$ and $15 \mathrm{~s}$ were applied before the acquisition for the observing time evolution of polarization distribution.

The experiment detecting ${ }^{19} \mathrm{~F}$ was performed with a static magnetic field of $0.7559 \mathrm{mT}$ (using a current of $1.937 \mathrm{~A})$. After delay times of $2,4,6$, or $8 \mathrm{~s}$, a $\pi / 2$ pulse was applied, followed by ten second acquisition. The raw data were two-fold zero filled, Fourier transformed and phased. Data were processed using Matlab (Mathworks, Natick, MA). To obtain a phased, real-valued spectrum, the FID was multiplied with with $\cos \left(2 \pi f_{0} t\right)-i \sin \left(2 \pi f_{0} t\right)$, where $f_{0}=31200 \mathrm{~Hz}$ is an arbitrarily chosen reference frequency. The resulting complex valued FID was multiplied with $\exp \left(i \cdot\left(\varphi_{1}+\varphi_{2} t\right)\right)$, where $\varphi_{1}$ and $\varphi_{2}$ are phasing factors, and was subsequently Fourier transformed.

Optical Measurements. The concentration of TEMPOL in a sample after its injection into the cell was determined from laser measurement of absorption $A=-\log _{10}\left(U_{\text {sample }}-U_{\text {dark }}\right) /\left(U_{\text {blank }}-U_{\text {dark }}\right)$, where $U_{\text {sample }}, U_{\text {blank }}$ and $U_{\text {dark }}$ are the output voltages observed in the output of the transimpedance amplifier proportional to the intensities of light in the presence of a DNP sample, without a DNP sample, and light blocked, respectively. The TEMPOL concentrations were calculated using $c=A / k$, where $k=0.0669 \mathrm{M}^{-1}$ was determined using solutions of known concentration in a spectrophotometer (BioSpec-mini, Shimadzu, Columbia, MD). The dilution factor of the sample during dissolution $d$ was then found from these concentrations as $d=c / c D N P$, where $c_{D N P}$ is the concentration of TEMPOL in 
the sample during DNP polarization known from sample preparation. The fraction of recovered sample was calculated as $r=V_{\text {cell }} \cdot d / V_{\mathrm{DNP}}$, where $V_{\mathrm{DNP}}$ is the volume of the DNP polarized sample aliquot.

Determination of Polarization Level. Using theory developed in [27], we calculated bulk magnetization level from the measured voltage signal $U_{\text {observed }}$ as

$$
M_{\mathrm{bulk}}=\frac{U_{\text {observed }}}{\left(2 \pi^{2} f\right) \cdot \mu_{0} \cdot w \cdot a_{c}^{2} \cdot Q \cdot g \cdot \sin (\theta)}
$$

where $f$ is the Larmor frequency, $\mu_{0}=4 \pi \cdot 10^{-7} \mathrm{H} \cdot \mathrm{m}^{-1}$ is the vacuum permeability, $w=242$ is the number of turns in the detection coil, $a_{c}=4.41 \cdot 10^{-3} \mathrm{~m}$ is the radius of the sample, $Q=29$ is the quality factor of the resonator and $\theta=\pi / 50.5$ is the flip angle of the excitation pulse. The preamplifier gain was $g=500 \cdot 0.896$ (set to 500 , reduced by 0.896 due to band-pass filtering). $U_{\text {observerd }}$ was calculated using two separate methods, first by taking the highest point from time domain data and second using the integral of the spectrum. In both cases, the ringdown signal was subtracted from the actual signal. The polarization level of the sample in the measurement cell was then calculated using

$$
P_{\mathrm{bulk}}=\frac{M_{\mathrm{bulk}}}{n \cdot \mu_{I}}
$$

where $n=5.814 \cdot 10^{28} \mathrm{~m}^{-3}$ is the number density of protons and $\mu_{I}=1.4106 \cdot 10^{-26} \mathrm{~J} \cdot \mathrm{T}^{-1}$ is the magnetic moment of the proton spin. Then the polarization level of spins before injection can be expressed as

$$
P_{\mathrm{DNP}}=P_{\mathrm{bulk}} / d
$$

\section{Results and Discussion}

Figures $2 \mathrm{a}$ and $\mathrm{b}$ illustrate very large enhancement of the LF NMR signal by D-DNP: the signal from a 160-fold diluted DNP sample in the cell tipped at a flip angle of $\pi / 50.5$ is still much larger than 
the signal of the thermally polarized sample tipped by the $\pi / 2$ flip angle. Because the D-DNP sample is hyperpolarized outside of the spectrometer, the optimization of transfer of the sample into the measurement cell is important. To this end, the time dependence of NMR signals obtained from the flow cell during the injection of the hyperpolarized sample was investigated (the upper panel of Figure 2c). It can be seen that the sample magnetization gradually increases and decreases as the hyperpolarized bolus flows through the cell. To monitor the concentration of radicals and dynamics of injection, an optical extinction measurement was also performed (the lower panel of Figure 2c) at the same time with the NMR measurement. The optical extinction indicated scattering in the sample caused by transiently appearing gas bubbles due to the use of pressurized gas for sample injection; however, after the sample had become stationary, its concentration was determined with good accuracy. NMR spectroscopy, on the other hand, was not affected by gas bubbles. Since the NMR signal was acquired for the entire duration of the experiment, signals from the pulse itself, as well as from the ringdown immediately following the pulse, are present in the data. These signals can be seen during the first $2 \mathrm{~ms}$ of the scan without sample (dark gray trace) in Figure 2. They can be removed from the data by subtraction of the background scan.

The signal from the hyperpolarized sample showed a shorter life time than the signal from thermal polarization (Figure $2 \mathrm{a}$ and $\mathrm{b}$ ). Shortening of the signal life time in the hyperpolarized sample occurs due to radiation damping because of the large spin magnetization [29]. A signal that was acquired without using the tuning capacitor, in order to avoid radiation damping, had a much longer decay time (light gray trace in Figure 2a). 

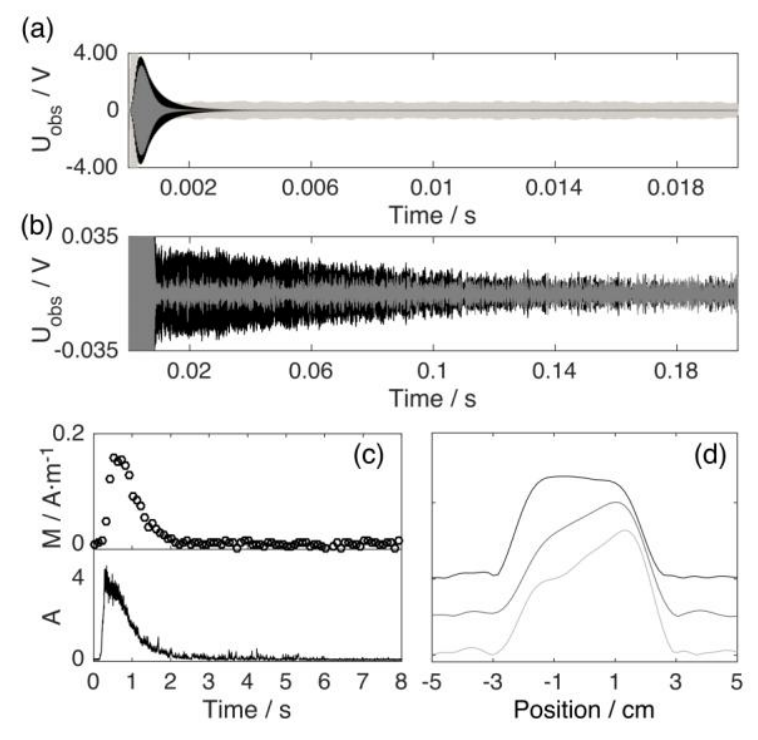

Figure 2: a) A single scan free induction decay (FID) from hyperpolarized water (15 mM TEMPOL during DNP polarization) excited with a $\pi / 50.5$ pulse and acquired with tuning capacitor (black curve) or without capacitor (light gray curve, enlarged by 29 times in the y axis in order to compensate for the $Q$ factor); b) FIDs averaged 2048 times from thermally polarized sample at $B_{0}=0.73281 \mathrm{mT}$ excited with $\pi / 2$ pulses. Dark Gray data lines represent the signal from background and pulse voltages in the absence of sample. c) Upper panel: Spin magnetization obtained from successive excitations during injection of samples into the flow cell acquired with 15 successive $\pi / 100$ excitations in intervals of 0.1 s. Lower panel: Optical extinction A (see the definition in the text) measured during sample injection. d) Stopped-flow one-dimensional NMR images obtained along the cell that show the distribution of the polarization in the cell at different delay times, from bottom to top: $5 \mathrm{~s}, 10 \mathrm{~s}, 15 \mathrm{~s}$.

In addition to time-evolution measurements coming from the whole sample, the 1D distribution of polarized sample was measured using NMR with one constantly applied gradient along the axis of the cell (Figure 2d). It can be seen that the distribution of the polarized spins changed over a time of several seconds of sample injection, building up on one side of the cell and then spreading over the whole cell. More generally, this visualization can be used to study the dynamics of fluid motion in the cell resulting from high pressure injection.

From the analysis of the dynamics, we found that the sample volume yielding the largest NMR signal can be captured in the flow cell by switching the injection valve after the optimal time determined from Figure 2c. Switching the injection valve pinches the flow at the inlet and the outlet of 
the cell, making the sample stationary. This stopped-flow method can be used to characterize the hyperpolarized sample in terms of NMR signal frequencies, relaxation rates or other observable parameters. A sequence of NMR signals obtained from multiple small flip angle excitations can for example be used for measuring spin-lattice relaxation time [30]. Time-domain data, as well as integrals of the Fourier transformed NMR signals obtained from such a sequence, are shown in the panels of Figures $3 \mathrm{a}$ and $\mathrm{b}$, respectively.
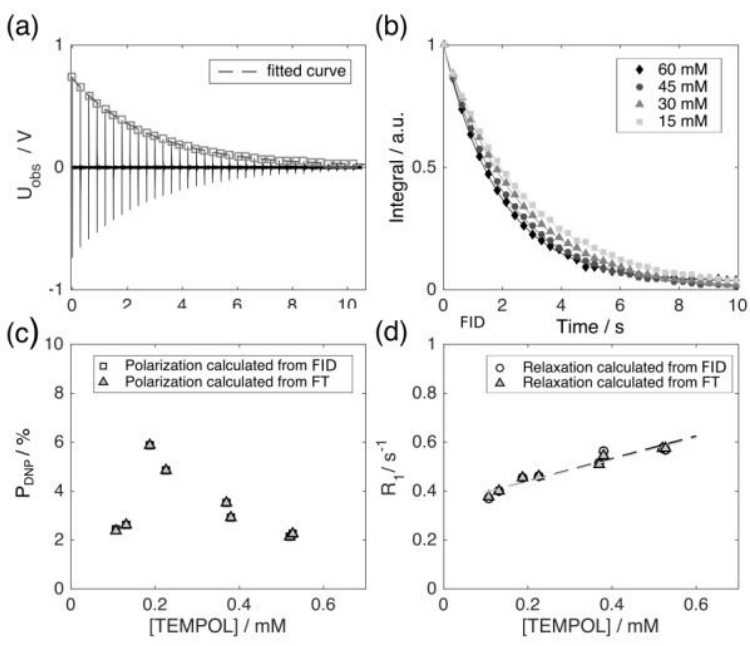

Figure 3: a) NMR time domain signal measured by successive $\pi / 50.5$ pulses applied to the DNP hyperpolarized sample with $15 \mathrm{mM}$ TEMPOL. Open squares indicate the maximum observed voltage from each scan. The electrical signal from rf pulses is removed by subtracting a scan when no sample was injected. b) integrated peak areas of the signal spectra obtained from hyperpolarized samples with different TEMPOL concentrations for measurement of spin-lattice relaxation time. Shown in the legend are TEMPOL concentrations during hyperpolarization, i.e. before dilution. c) Polarization of hyperpolarized sample as a function of TEMPOL concentration. d) Measured spin-lattice relaxation time as a function of the TEMPOL concentration as determined by the optical measurement.

From a fit of the time-domain signal (Figure 3a; the amplitude of $0.402 \mathrm{~V}$ corresponds to a bulk magnetization of $0.186 \mathrm{~A} \cdot \mathrm{m}^{-1}$ or a bulk polarization of $2.26 \cdot 10^{-4}$ ) or from the integrated peak areas in the spectrum (Figure 3b), the initial signal amplitude can be found, allowing for the determination of bulk magnetization and polarization according to Eq. $2-3$. The resulting polarization values obtained 
at different radical concentrations are listed in Table 1. For comparison, data from the integrated area of the spectrum from Figure 2b's FID, the bulk polarization for water that has been thermally polarized in the measurement field was determined to be $2.41 \cdot 10^{-9}$, which is in good agreement with the expected value of $2.50 \cdot 10^{-9}$ at $\mathrm{T}=300 \mathrm{~K}$. Consequently, the hyperpolarization leads to a bulk signal enhancement of $9.4 \cdot 10^{4}$ even after the dilution of the originally polarized aliquot.

Table 1. Calculated polarization levels and related data. Values in columns are: $c_{D N P}$ - radical concentration in hyperpolarized aliquot, $c$ - radical concentration in cell calculated from optical signal, $U_{\text {observed }}$-voltage observed from photodiode, $M_{\text {bulk }}$ - bulk magnetization calculated based Eq. 2, $P_{D N P}$ - polarization level calculated based on Eq. 3. FID and Fourier Transform data gave essentially the same results.

\begin{tabular}{llllll}
\hline$c_{\mathrm{DNP}}[\mathrm{mM}]$ & $c[\mathrm{mM}]$ & $\begin{array}{l}U_{\text {observed }}[\mathrm{V}] \\
(\text { FID })\end{array}$ & $\begin{array}{l}U_{\text {observed }}[\mathrm{V}] \\
\text { (from FT) }\end{array}$ & $\begin{array}{l}M_{\text {bulk }} \\
\left.{ }^{1}\right](\text { from FT) }\end{array}$ & \begin{tabular}{l} 
FT) \\
\hline
\end{tabular} \\
\hline 15 & 0.130 & 0.402 & 0.403 & 0.187 & 2.64 \\
15 & 0.107 & 0.304 & 0.302 & 0.140 & 2.39 \\
30 & 0.187 & 0.646 & 0.649 & 0.300 & 5.90 \\
30 & 0.227 & 0.648 & 0.651 & 0.301 & 4.85 \\
45 & 0.379 & 0.434 & 0.435 & 0.201 & 2.93 \\
45 & 0.370 & 0.514 & 0.514 & 0.238 & 3.54 \\
60 & 0.519 & 0.323 & 0.326 & 0.151 & 2.14 \\
60 & 0.526 & 0.352 & 0.351 & 0.163 & 2.26 \\
\hline
\end{tabular}

Since this data stems from hyperpolarized water, which has been further diluted with unpolarized water during sample injection, bulk polarization as discussed above represents a reasonable parameter to characterize the signal intensity. However, the D-DNP experiment allows hyperpolarization of a large number of different substances, not necessarily identical to the solvent used for dissolution, and with particularly large signal enhancement, it becomes possible to study highly dilute analytes by LF NMR. Therefore it is of interest to consider the polarization level in the flow cell of the originally 
hyperpolarized sample material. This polarization level can be calculated from the bulk magnetization using the dilution factor determined by an optical measurement of TEMPOL concentration of the stationary sample in the flow cell. For the data from Figure 3a, the polarization level of the originally hyperpolarized component $\left(P_{\mathrm{DNP}}\right)$ was $2.40 \%$, whereas at optimal radical concentration, it was increased to close to $6 \%$ (Table 1).

(a)

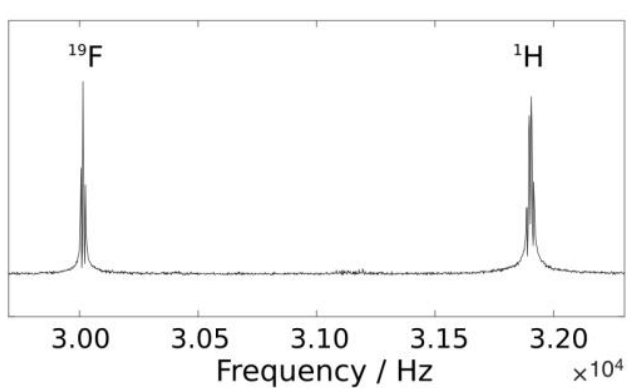

(b)

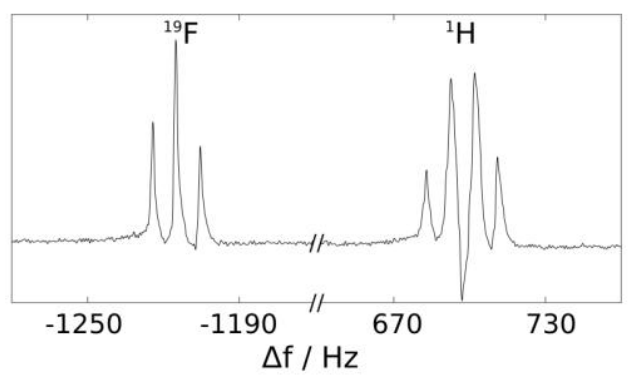

(c)

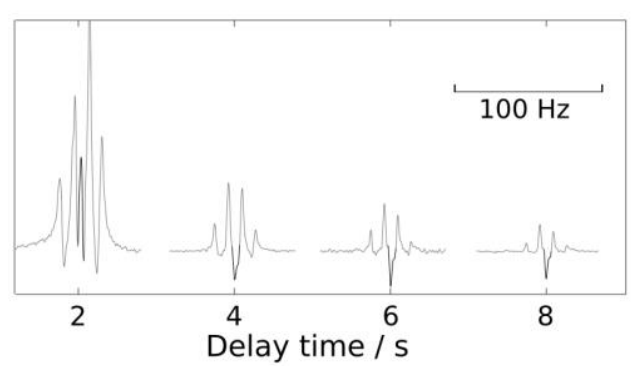

Figure 4: a) Magnitude spectrum of hyperpolarized trifluoroethanol (TFE; $\left.\mathrm{CF}_{3} \mathrm{CH}_{2} \mathrm{OH}\right)$ with a delay time of $4 \mathrm{~s}$ after injection into the low-field NMR using pressurized water. b) Phased real spectrum, shown with offset $\Delta$ f from $31,200 \mathrm{~Hz}$. The actual frequency for the center of the ${ }^{19} \mathrm{~F}$ multiplet is 31,200 $1185=30,015 \mathrm{~Hz}$, and for the ${ }^{1} \mathrm{H}$ multiplet is $31,200+703=31,903 \mathrm{~Hz} . \mathrm{c}$ ) Enlarged spectral region corresponding to ${ }^{1} \mathrm{H}$ signal, from separate measurements measured at different delay times. The central line corresponding to protons without observable J-coupling is highlighted with dark color. 
In addition to the initial signal intensity, the same data allow the quantification of paramagnetic relaxation enhancement (PRE) under the low-field measurement conditions. A plot of the relaxation rates obtained at different radical concentrations present in the cell after dilution of the sample is shown in Figure 3d. The resulting fitted line gives a relaxivity of $\varepsilon=0.45 \mathrm{~s}^{-1} \mathrm{mM}^{-1}$ under the conditions of measurement field of $0.7 \mathrm{mT}$, which is significantly larger than at high field, $\varepsilon=0.133 \mathrm{~s}^{-1} \mathrm{mM}^{-1}$ at $300 \mathrm{~K}$. The measurement field employed here corresponds closely to that encountered in the injection path between a DNP polarizer and a high-field NMR magnet [31]. The low-field $R_{1}$ measurement can therefore serve as an indicator of signal loss due to relaxation during sample injection in a D-DNP experiment and can be used for the optimization of polarization transfer. Comparison of the ${ }^{1} \mathrm{H}$ data obtained here with ${ }^{13} \mathrm{C}$ relaxation data measured by Miéville et al. [31] suggests that low-field relaxation plays an even more important role in the case of dissolution DNP experiments using ${ }^{1} \mathrm{H}$.

The data in Figure $3 \mathrm{c}$ most interestingly illustrate substantial polarization gain at the optimal radical concentration, arising from a combination of solid state polarization efficiency and losses during sample injection. It can be also noted that under the conditions yielding highest polarization (30 $\mathrm{mM}$ during polarization, resulting in $0.2 \mathrm{mM}$ after dissolution), the paramagnetic contribution to the overall relaxation rate of $R_{1}=0.46 \mathrm{~s}^{-1}$ is still modest.

In addition to the determination of $T_{1}$ relaxation and its effect on polarization of samples in the DDNP experiment, LF NMR permits the study of other phenomena such as cross-relaxation. The large spin polarization produced by hyperpolarization can significantly enhance otherwise weak signals by means of the nuclear Overhauser effect [32,33]. In high-field NMR, in particular for the determination of heteronuclear cross-relaxation, this measurement however requires specialized multi-channel detectors [34]. An interesting feature of LF NMR in this regard is the dramatically reduced frequency bandwidth, resulting in the ability to obtain signals from distinct nuclei in the same spectrum. In Figure 4, the signal evolution due to $\left\{{ }^{1} \mathrm{H},{ }^{19} \mathrm{~F}\right\}$-NOE effect is observed after injection of hyperpolarized 2,2,2- 
Trifluoroethanol (TFE) mixed with water. Although microwave frequency was adjusted for optimal ${ }^{19} \mathrm{~F}$ polarization, the presence of ${ }^{1} \mathrm{H}$ polarization is also expected. Signals at the frequency of both nuclei are observable (Figure 4a) and are split with the respective $J$-coupling. The multiplet structures are a triplet for the $\mathrm{CF}_{3}$ group of TFE coupled to $\mathrm{CH}_{2}$, a quartet for the $\mathrm{CH}_{2}$ group of TFE coupled to $\mathrm{CF}_{3}$, and a singlet for the $\mathrm{OH}$ groups of TFE and water. Although in a spectrum acquired shortly after injection, hyperpolarized signals from all components are observed, the singlet signal becomes inverted at later time (Figure $4 \mathrm{~b}$ ). This behavior is explained by the NOE from hyperpolarized ${ }^{19} \mathrm{~F}$ and ${ }^{1} \mathrm{H}$ spins of the $\mathrm{CF}_{3}$ and $\mathrm{CH}_{2}$ groups of TFE. Using D-DNP hyperpolarized NMR therefore allows the direct observation of NOE buildup curves at low field, which would otherwise be subject to prohibitive sensitivity limits. Such curves may in the future be used for determining the dynamics of intermolecular interactions and other physical properties of solvent-solute mixtures. It is also possible to use the para-hydrogen polarization or Overhauser DNP methods to directly hyperpolarize fluorine and protons at low field $[35,36]$. The D-DNP method shown here is complementary to these methods and yields different results. For example, typical Overhauser DNP enhancements are on the order of $10^{3} \sim 10^{4}$. On the other hand, D-DNP enhancements readily reach $10^{4} \sim 10^{5}$, albeit requiring more instrumentation and including some constraints on sample size or concentration.

\section{Conclusions}

In summary, we have described a low-field NMR experiment capable of characterizing compounds hyperpolarized by D-DNP. A combination of NMR signal acquisition with in situ concentration determination by laser based spectrophotometry allowed quantification of spin-lattice relaxation properties in relationship with the concentration of the free radical employed for DNP hyperpolarization. Since these relaxation rates contribute to the losses of hyperpolarization during 
sample injection in typical D-DNP experiments, the present measurement provides a basis for optimizing the high-field D-DNP experiment. Further, the nuclear Overhauser effect at low field can readily be observed between different nuclei or between the same nuclei with different coupling multiplicity. This observation opens a new NMR frequency regime for the determination of the dynamics of intermolecular interactions by cross-relaxation.

\section{Acknowledgments}

Financial support from the National Science Foundation (Grants CHE-1404548 and CHE1404529) and the Welch Foundation (Grant A-1658) is gratefully acknowledged. 


\section{References}

[1] M. Espy, M. Flynn, J. Gomez, C. Hanson, R. Kraus, P. Magnelind, K. Maskaly, A. Matlashov, S. Newman, M. Peters, H. Sandin, I. Savukov, L. Schultz, A. Urbaitis, P. Volegov, V. Zotev, Applications of Ultra-Low Field Magnetic Resonance for Imaging and Materials Studies, IEEE Trans. Appl. Supercond. 19 (2009) 835-838. doi:10.1109/TASC.2009.2018517.

[2] D.M. Korzhnev, L.E. Kay, Probing Invisible, Low-Populated States of Protein Molecules by Relaxation Dispersion NMR Spectroscopy: An Application to Protein Folding, Acc. Chem. Res. 41 (2008) 442-451. doi:10.1021/ar700189y.

[3] N.R. Skrynnikov, F.A.A. Mulder, B. Hon, F.W. Dahlquist, L.E. Kay, Probing Slow Time Scale Dynamics at Methyl-Containing Side Chains in Proteins by Relaxation Dispersion NMR Measurements: Application to Methionine Residues in a Cavity Mutant of T4 Lysozyme, J. Am. Chem. Soc. 123 (2001) 4556-4566. doi:10.1021/ja004179p.

[4] F.A.A. Mulder, N.R. Skrynnikov, B. Hon, F.W. Dahlquist, L.E. Kay, Measurement of Slow ( $\mu \mathrm{s}-\mathrm{ms}$ ) Time Scale Dynamics in Protein Side Chains by 15N Relaxation Dispersion NMR Spectroscopy: Application to Asn and Gln Residues in a Cavity Mutant of T4 Lysozyme, J. Am. Chem. Soc. 123 (2001) 967-975. doi:10.1021/ja003447g.

[5] P.T. Callaghan, C.D. Eccles, J.D. Seymour, An earth's field nuclear magnetic resonance apparatus suitable for pulsed gradient spin echo measurements of self-diffusion under Antarctic conditions, Rev. Sci. Instrum. 68 (1997) 4263. doi:10.1063/1.1148340.

[6] P.. Callaghan, R. Dykstra, C.. Eccles, T.. Haskell, J.. Seymour, A nuclear magnetic resonance study of Antarctic sea ice brine diffusivity, Cold Reg. Sci. Technol. 29 (1999) 153-171. doi:10.1016/S0165-232X(99)00024-5.

[7] R. McDermott, A.H. Trabesinger, M. Mück, E.L. Hahn, A. Pines, J. Clarke, Liquid-State NMR and Scalar Couplings in Microtesla Magnetic Fields, Science. 295 (2002) 2247-2249. doi:10.1126/science.1069280.

[8] S. Appelt, F.W. Häsing, H. Kühn, U. Sieling, B. Blümich, Analysis of molecular structures by homo- and hetero-nuclear J-coupled NMR in ultra-low field, Chem. Phys. Lett. 440 (2007) 308312. doi:10.1016/j.cplett.2007.03.096.

[9] S. Appelt, H. Kühn, F.W. Häsing, B. Blümich, Chemical analysis by ultrahigh-resolution nuclear magnetic resonance in the Earth's magnetic field, Nat. Phys. 2 (2006) 105-109. doi:10.1038/nphys211.

[10] M.P. Ledbetter, C.W. Crawford, A. Pines, D.E. Wemmer, S. Knappe, J. Kitching, D. Budker, Optical detection of NMR J-spectra at zero magnetic field, J. Magn. Reson. 199 (2009) 25-29. doi:10.1016/j.jmr.2009.03.008.

[11] J.W. Blanchard, M.P. Ledbetter, T. Theis, M.C. Butler, D. Budker, A. Pines, High-Resolution Zero-Field NMR J-Spectroscopy of Aromatic Compounds, J. Am. Chem. Soc. 135 (2013) 36073612. doi:10.1021/ja312239v.

[12] D.I. Hoult, B. Bhakar, NMR signal reception: Virtual photons and coherent spontaneous emission, (n.d.). http://moodle.technion.ac.il/pluginfile.php/531078/mod_resource/content/1/Hoult.pdf (accessed August 29, 2015).

[13] S. Xu, V.V. Yashchuk, M.H. Donaldson, S.M. Rochester, D. Budker, A. Pines, Magnetic resonance imaging with an optical atomic magnetometer, Proc. Natl. Acad. Sci. 103 (2006) 12668-12671. doi:10.1073/pnas.0605396103. 
[14] D. Budker, M. Romalis, Optical magnetometry, Nat. Phys. 3 (2007) 227-234. doi:10.1038/nphys566.

[15] I.K. Kominis, T.W. Kornack, J.C. Allred, M.V. Romalis, A subfemtotesla multichannel atomic magnetometer, Nature. 422 (2003) 596-599. doi:10.1038/nature01484.

[16] I.M. Savukov, M.V. Romalis, NMR Detection with an Atomic Magnetometer, Phys. Rev. Lett. 94 (2005) 123001. doi:10.1103/PhysRevLett.94.123001.

[17] A.N. Matlachov, P.L. Volegov, M.A. Espy, J.S. George, R.H. Kraus Jr., SQUID detected NMR in microtesla magnetic fields, J. Magn. Reson. 170 (2004) 1-7. doi:10.1016/j.jmr.2004.05.015.

[18] A.M. Coffey, M.L. Truong, E.Y. Chekmenev, Low-field MRI can be more sensitive than highfield MRI, J. Magn. Reson. 237 (2013) 169-174. doi:10.1016/j.jmr.2013.10.013.

[19] W. Zhang, C.I. Ratcliffe, I.L. Moudrakovski, C.-Y. Mou, J.A. Ripmeester, Distribution of Gallium Nanocrystals in Ga/MCM-41 Mesocomposites by Continuous-Flow Hyperpolarized 129Xe NMR Spectroscopy, Anal. Chem. 77 (2005) 3379-3382. doi:10.1021/ac050076j.

[20] V.S. Zotev, T. Owens, A.N. Matlashov, I.M. Savukov, J.J. Gomez, M.A. Espy, Microtesla MRI with dynamic nuclear polarization, J. Magn. Reson. 207 (2010) 78-88. doi:10.1016/j.jmr.2010.08.015.

[21] B.D. Armstrong, S. Han, Overhauser Dynamic Nuclear Polarization To Study Local Water Dynamics, J. Am. Chem. Soc. 131 (2009) 4641-4647. doi:10.1021/ja809259q.

[22] W. Müller-Warmuth, K. Meise-Gresch, Molecular motions and interactions as studied by dynamic nuclear polarization (DNP) in free radical solutions, Adv. Magn. Reson. 11 (1983) 1-45.

[23] M. Bennati, C. Luchinat, G. Parigi, M.-T. Türke, Water 1H relaxation dispersion analysis on a nitroxide radical provides information on the maximal signal enhancement in Overhauser dynamic nuclear polarization experiments, Phys. Chem. Chem. Phys. 12 (2010) 5902. doi:10.1039/c002304n.

[24] M. Sarracanie, B.D. Armstrong, J. Stockmann, M.S. Rosen, High speed 3D overhauser-enhanced MRI using combined b-SSFP and compressed sensing, Magn. Reson. Med. 71 (2014) 735-745. doi:10.1002/mrm.24705.

[25] G. Gottardi, P. Mesirca, C. Agostini, D. Remondini, F. Bersani, A four coil exposure system (tetracoil) producing a highly uniform magnetic field, Bioelectromagnetics. 24 (2003) 125-133. doi:10.1002/bem.10074.

[26] S. Bowen, C. Hilty, Rapid sample injection for hyperpolarized NMR spectroscopy, Phys. Chem. Chem. Phys. 12 (2010) 5766-5770. doi:10.1039/C002316G.

[27] I.M. Savukov, H.-Y. Chen, T. Karaulanov, C. Hilty, Method for accurate measurements of nuclearspin optical rotation for applications in correlated optical-NMR spectroscopy, J. Magn. Reson. 232 (2013) 31-38. doi:10.1016/j.jmr.2013.04.008.

[28] R. Kaptein, K. Dijkstra, C.E. Tarr, A single-scan fourier transform method for measuring spinlattice relaxation times, J. Magn. Reson. 1969. 24 (1976) 295-300. doi:10.1016/00222364(76)90039-1.

[29] N. Bloembergen, R.V. Pound, Radiation Damping in Magnetic Resonance Experiments, Phys. Rev. 95 (1954) 8-12. doi:10.1103/PhysRev.95.8.

[30] R. Freeman, H.D.W. Hill, Fourier Transform Study of NMR Spin-Lattice Relaxation by "Progressive Saturation," J. Chem. Phys. 54 (1971) 3367-3377. doi:10.1063/1.1675352.

[31] P. Miéville, S. Jannin, G. Bodenhausen, Relaxometry of insensitive nuclei: Optimizing dissolution dynamic nuclear polarization, J. Magn. Reson. 210 (2011) 137-140. doi:10.1016/j.jmr.2011.02.006. 
[32] Y.-Q. Song, Spin polarization-induced nuclear Overhauser effect: An application of spinpolarized xenon and helium, Concepts Magn. Reson. 12 (2000) 6-20. doi:10.1002/(SICI)10990534(2000)12:1<6::AID-CMR3>3.0.CO;2-U.

[33] G. Navon, Y.-Q. Song, T. Room, S. Appelt, et al, Enhancement of solution NMR and MRI with laser-polarized xenon, Science. 271 (1996) 1848.

[34] J. Kim, M. Liu, H.-Y. Chen, C. Hilty, Determination of Intermolecular Interactions Using Polarization Compensated Heteronuclear Overhauser Effect of Hyperpolarized Spins, Anal. Chem. 87 (2015) 10982-10987. doi:10.1021/acs.analchem.5b02934.

[35] M.E. Halse, P.T. Callaghan, A dynamic nuclear polarization strategy for multi-dimensional Earth's field NMR spectroscopy, J. Magn. Reson. 195 (2008) 162-168. doi:10.1016/j.jmr.2008.09.007.

[36] B.C. Hamans, A. Andreychenko, A. Heerschap, S.S. Wijmenga, M. Tessari, NMR at earth's magnetic field using para-hydrogen induced polarization, J. Magn. Reson. 212 (2011) 224-228. doi:10.1016/j.jmr.2011.06.011. 